\title{
Saúde, dor e atividades de vida diária entre idosos praticantes de Lian Gong e sedentários
}

\author{
Health, pain and daily activities among elderly people \\ practicing Lian Gong and sedentary elderly people
}

\author{
Vanessa Cristina Dias Bobbo ${ }^{1}$ \\ Danilo Donizetti Trevisan ${ }^{1}$ \\ Maria Clara Estanislau do Amaral ${ }^{1}$ \\ Eliete Maria Silva ${ }^{1}$
}

${ }^{1}$ Faculdade de Enfermagem, Universidade Estadual de Campinas. R. Tessália Vieira de Camargo 126, Cidade Universitária Zeferino Vaz 13083-887 Campinas SP Brasil.vanessacdbobbo@ gmail.com

\begin{abstract}
It is a well-known fact that the practice of physical activity on a regular basis among elderly people contributes to the prevention and reduction of pain, as well as promote active ageing. Based on this premise, the scope of this study was to evaluate the presence of chronic pain among elderly people attended in a primary health care unit in a city in the interior of Sao Paulo State, Brazil, by comparing those who practice Chinese gymnastics (Lian Gong) and the sedentary. Sixty elderly people were divided between 30 sedentary and 30 who participate in a group practicing Lian Gong. The presence of pain was assessed through the Nordic Questionnaire on Musculoskeletal Symptoms, already adapted and validated for Brazilian culture. When questioned about pain in the last seven days, the Lian Gong group showed a higher presence $(62,3 \%)$ of pain when compared to the sedentary group, although they had fewer difficulties in performing daily activities (55,5\%). Among the active elderly the presence of lower back pain was statistically inferior $(p<0.05)$ when comparing the groups. The practice of Lian Gong was associated to a more positive perception of one's health, less use of medicines, as well as the adopting of self-care practices and the feeling of fewer impairments in performing daily activities. Key words Exercise, Chronic pain, Complementary therapies, Primary health care
\end{abstract}

Resumo A prática regular de atividade física entre idosos contribui tanto para a prevenção e a redução da dor, quanto atua na promoção do envelhecimento ativo. Diante disso, este estudo teve como objetivo avaliar a presença de dor crônica em idosos atendidos em uma unidade de atenção primária do interior do estado de São Paulo, comparando os praticantes da ginástica chinesa (Lian Gong) e os idosos sedentários. Participaram deste estudo 60 idosos, sendo 30 integrantes de um grupo de Lian Gong e 30 sedentários. A presença da dor foi avaliada pelo Questionário Nórdico de Sintomas Osteomusculares, adaptado e validado para a cultura brasileira. A presença da dor nos últimos sete dias foi maior (62,3\%) no grupo de praticantes de Lian Gong, porém com menor impedimento para realização de atividades (55,5\%) quando comparados com o grupo de sedentários. Nos indivíduos ativos a presença da dor na parte inferior das costas foi estatisticamente menor ( $p$ $<0,05)$ quando comparados ambos os grupos. A prática do Lian Gong esteve relacionada com a percepção positiva da própria saúde, o menor uso de medicamentos, a adoção de práticas de autonomia no próprio cuidado e a sensação de menor impedimento para realizar atividades de vida diária. Palavras-chave Exercício, Dor crônica, Terapias complementares, Atenção Primária à Saúde 


\section{Introdução}

O envelhecimento populacional é assunto que gera importantes discussões nos campos econômico, social e da saúde. Segundo projeções das Nações Unidas, a porcentagem de pessoas com 60 anos ou mais no Brasil aumentará de 12\%, em 2015, pra 29\%, em 2050. Atribui-se o aumento da longevidade, principalmente, aos avanços no campo da saúde pública, da medicina e à melhoria nas condições de vida da população ${ }^{1}$. Entretanto, pode-se observar que esse aumento está fortemente associado a uma crescente taxa de doenças crônicas não transmissíveis (DCNT) e seus agravos mais prevalentes ${ }^{1,2}$.

As condições crônicas apresentadas por indivíduos idosos são predominantemente associadas à dor ${ }^{3}$. As DCNT que têm como sintoma comum a dor, como as doenças osteoarticulares, devem ser tratadas como problema de saúde coletiva, pois são particularmente prevalentes na população idosa brasileira. A abordagem das políticas de saúde do idoso deve contemplar o desenvolvimento de estratégias objetivas, como a redução da obesidade e o desenvolvimento de práticas regulares de atividade física ${ }^{4}$.

As ações que visam prevenir e reduzir a dor corporal crônica são consideradas importantes estratégias para a manutenção das atividades cotidianas e a autonomia dos idosos. A prática regular de atividade física não somente contribui para a prevenção e redução da dor, como também promove o envelhecimento ativo. Um bom exemplo são as atividades de grupo, que ademais proporcionam socialização e lazer, como o Lian Gong 5 .

O Lian Gong consiste em um conjunto de exercícios, organizados em séries, que agem na prevenção e no tratamento de dores e de doenças crônicas. Trata-se de uma técnica terapêutica criada na China pelo médico Zhuang Yuen Ming, que buscou unir a terapia ao fortalecimento harmonioso do corpo. A combinação entre exercício, persistência e habilidade tem raízes na cultura chinesa, baseado em terapias e movimentos da medicina tradicional, das artes tradicionais da guerra e de antigos exercícios terapêuticos. Esta terapia trabalha principalmente sobre pescoço, ombros, cintura e pernas, e tem percorrido vários países, sobretudo Japão, Indonésia e Estados Unidos da América. No Brasil, a repercussão da introdução do Lian Gong mostrou que esta técnica tem enriquecido as práticas integrativas desenvolvidas no sistema público de saúde, contribuindo para a qualidade de vida, sobretudo dos idosos ${ }^{6}$.
Em bases de dados nacionais e internacionais, pouco se tem explorado e publicado acerca dos efeitos da prática do Lian Gong entre idosos e sua relação com o tratamento da dor corporal crônica. A partir dos resultados de estudos anteriores $^{3,5}$, há indícios de que a prática do Lian Gong promove saúde e bem estar entre indivíduos idosos, reduzindo agravos relacionados a problemas crônicos. Como terapia que se presta à promoção e aos cuidados de saúde num nível coletivo, reduzem-se os gastos em saúde cujo foco da assistência é a atenção primária. Esta é uma abordagem relevante, sobretudo em países com economia em desenvolvimento, como o Brasil, que apresentam uma crescente população de idosos que demanda prevenção e tratamento de problemas crônicos. Tendo em vista essas considerações, este estudo tem como objetivo avaliar a presença de dor crônica em idosos atendidos em uma unidade de atenção primária do interior do estado de São Paulo, comparando os praticantes da ginástica chinesa (Lian Gong) e os idosos sedentários.

\section{Métodos}

Trata-se de um estudo transversal, com abordagem comparativa, sobre dados coletados no período de agosto a novembro de 2012, em uma unidade de atenção primária à saúde, no município de Campinas- SP. Esta unidade faz parte do Sistema Único de Saúde e é composta por quatro equipes da Estratégia Saúde da Família.

Desde agosto de 2001, esta unidade de saúde desenvolve práticas de Lian Gong em grupo, reunidos três vezes na semana. No período da coleta de dados, 36 indivíduos participavam regularmente dele há mais de seis meses, com predomínio de idosos. Os integrantes do grupo que atenderam aos critérios de inclusão e aceitaram participar (30 indivíduos ativos) compuseram a amostra do estudo.

Foram incluídos indivíduos com idade igual ou maior que 60 anos, de ambos os sexos, com habilidade para comunicar-se em português do Brasil e compreender as questões dos instrumentos aplicados. A partir do número de indivíduos ativos (praticantes de Lian Gong) disponível, constitui-se a composição esperada do grupo de sedentários, para comparação das duas amostras. Estabeleceu-se que se considerariam sedentários os idosos que refeririam não praticar qualquer atividade física regular. Para compor este segundo grupo de 30 idosos sedentários, foram convi- 
dados os indivíduos que compareciam à unidade de saúde para atendimento, fosse ele agendado ou não, e atendiam aos critérios de inclusão previamente descritos, numa amostragem por conveniência.

Em ambos os grupos, aplicou-se um instrumento de caracterização do idoso adaptado da caderneta de saúde da pessoa idosa, do Ministério da Saúde ${ }^{7}$. O questionário nórdico de sintomas osteomusculares, instrumento escolhido por sua facilidade da compreensão ${ }^{8}$, foi aplicado com o fim de caracterizar a dor corporal; este instrumento possibilita levantar as dores nos últimos doze meses e a impossibilidade de realizar alguma atividade física devido às dores relatadas. Além disso, aponta se os sujeitos pesquisados procuraram alguma unidade/profissional de saúde devido às dores e se apresentaram dor nos últimos sete dias prévios à aplicação do questionário.

Os medicamentos utilizados pelos idosos dos dois grupos foram categorizados no nível 1, segundo a Anatomical-Therapeutic-Chemical Classification - ATC da Organização Mundial da Saúde (OMS) ${ }^{9}$.

O presente estudo foi aprovado pelo Comitê de Ética em Pesquisa da Universidade Estadual de Campinas e conduzido de acordo com os princípios da resolução vigente à época. As entrevistas foram realizadas somente após os idosos serem orientados quanto aos objetivos da pesquisa e assinarem o Termo de Consentimento Livre e Esclarecido em duas vias.

\section{Análise estatística}

Os dados foram organizados em planilha do Microsoft Office Excel ${ }^{\circledR}$, versão 2010, usando dupla digitação. Foi aplicado o teste Qui-quadrado $\left(x^{2}\right)$ para variáveis qualitativas, a fim de detectar possíveis associações, e o teste de Mann Whitney nas variáveis quantitativas, para estabelecer comparações. Num primeiro momento, as análises foram feitas considerando os grupos sedentário e ativo. Posteriormente, foram estabelecidas associações levando em conta as respostas do total da amostra, de acordo com suas características. Para todas as análises usou-se o software estatístico SAS ${ }^{\circledR}$ (Statistical Analysis System), versão 9.2; o nível de significância adotado foi de 5\%.

\section{Resultados}

$\mathrm{Na}$ análise das informações dos 60 idosos pesquisados, a média de idade das pessoas do grupo dos ativos foi de 71,6 anos $( \pm 6,3)$ e a média dos sedentários foi de 70,2 anos $( \pm 5,3)$. Em ambos os grupos a maioria dos participantes era de mulheres, ativas $\mathrm{n}=30(100 \%)$ e sedentárias $\mathrm{n}=$ $25(83,3 \%)$. A maioria (60\%) dos entrevistados ativos classificou sua própria saúde como muito boa ou boa $(\mathrm{p}<0,05)$. A Tabela 1 apresenta a caracterização geral da amostra.

A Tabela 2 discrimina os dados relacionados aos agravos da saúde relatados pelos participantes. Em ambos os grupos, os problemas de saúde mais citados foram os cardiovasculares, metabólicos e os osteomusculares. Os indivíduos sedentários utilizavam em média 3,6 medicamentos/ dia, enquanto nos indivíduos praticantes de Lian Gong a média era de 2,4 medicamentos/dia. Os medicamentos de ação cardiovascular e metabólica foram os mais consumidos em ambos os grupos, seguidos dos medicamentos de ação sobre o aparelho digestivo e o metabolismo. A frequência de quedas entre os ativos foi maior ( $\mathrm{p}$-valor < $0,05)$.

Os dados obtidos por meio do questionário nórdico de sintomas osteomusculares apontaram que não houve diferença significativa nos dois grupos quanto à quantidade total de dor referida ou sua localização anatômica, conforme apresentado na Tabela 3. Contudo, para a região inferior das costas, a prática de Lian Gong esteve associada a uma menor sensação de restrição ao realizar atividades de vida diária $(\mathrm{p}<0,05)$. Em geral, o impedimento para realização de atividades devido à dor foi menor no grupo de praticantes de Lian Gong quando comparados com o grupo de sedentários.

Quando a dor foi discriminada nas várias regiões do corpo, constatou-se que 91,3\% dos indivíduos em união estável não apresentavam impedimento de movimentação do pescoço (p-valor = 0,0008); assim como 95,65\% destes indivíduos não apresentaram dores no cotovelo (p-valor = 0,03). Em relação aos participantes que passam o dia acompanhados, 97,14\% não apresentaram relatos de dores no cotovelo ( $\mathrm{p}$-valor $<0,0001$ ). Em contrapartida, 92\% dos indivíduos que passam o dia sozinhos apresentaram dor na parte inferior das costas ( $\mathrm{p}$-valor $=0,0146$ ).

\section{Discussão}

O presente estudo é um dos primeiros a avaliar a dor e sua relação com a prática do Lian Gong no Brasil. Observou-se que a maioria das idosas ativas era viúva, enquanto a maioria dos sedentários 
Tabela 1. Caracterização sociodemográfica dos indivíduos. Campinas, SP, 2012.

\begin{tabular}{|c|c|c|c|}
\hline Categorias & $\begin{array}{c}\text { Ativos } \\
\mathbf{n}(\%)\end{array}$ & $\begin{array}{c}\text { Sedentários } \\
\mathbf{n}(\%)\end{array}$ & $\begin{array}{r}\text { Total } \\
\text { n }(\%)\end{array}$ \\
\hline \multicolumn{4}{|l|}{ Estado civil } \\
\hline Casados & $9(30,0)$ & $14(46,6)$ & $23(38,3)$ \\
\hline Separados & $2(6,8)$ & $1(3,3)$ & $3(5)$ \\
\hline Solteiros & $2(6,8)$ & $5(16,6)$ & $7(11,7)$ \\
\hline Viúvos & $17(56,6)$ & $10(33,3)$ & $27(45)$ \\
\hline Total & $30(100)$ & $30(100)$ & $60(100)$ \\
\hline \multicolumn{4}{|l|}{ Ocupação atual } \\
\hline Sim & $10(33,3)$ & $8(26,6)$ & $18(30)$ \\
\hline Não & $20(66,6)$ & $22(73,3)$ & $42(70)$ \\
\hline Total & $30(100)$ & $30(100)$ & $60(100)$ \\
\hline \multicolumn{4}{|l|}{ Tipo de ocupação (última ou atual) } \\
\hline Não trabalhou & $0(0)$ & $1(3,3)$ & $1(1,7)$ \\
\hline Administrativo/atendimento público & $5(16,6)$ & $6(20)$ & $11(18,3)$ \\
\hline Indústria & $4(13,3)$ & $5(16,6)$ & $9(15)$ \\
\hline Serviços domésticos e alimentação & $8(26,6)$ & $9(30)$ & $17(28,4)$ \\
\hline Costureira & $9(30)$ & $2(6,6)$ & $11(18,3)$ \\
\hline Serviços diversos & $4(13,3)$ & $7(23,3)$ & $11(18,3)$ \\
\hline Total & $30(100)$ & $30(100)$ & $60(100)$ \\
\hline \multicolumn{4}{|l|}{ Aposentado(a) ou pensionista } \\
\hline Sim & $24(80)$ & $23(76,6)$ & $47(78,3)$ \\
\hline Não & $6(20)$ & $7(23,3)$ & $13(21,7)$ \\
\hline Total & $30(100)$ & $30(100)$ & $60(100)$ \\
\hline \multicolumn{4}{|l|}{ Mora sozinho } \\
\hline Sim & $7(23,3)$ & $5(16,6)$ & $12(20)$ \\
\hline Não & $23(76,6)$ & $25(83,3)$ & $48(80)$ \\
\hline Total & $30(100)$ & $30(100)$ & $60(100)$ \\
\hline \multicolumn{4}{|l|}{ Passa o dia sozinho } \\
\hline Sim & $14(46,6)$ & $11(36,6)$ & $25(41,7)$ \\
\hline Não & $16(53,3)$ & $19(63,3)$ & $35(58,3)$ \\
\hline Total & $30(100)$ & $30(100)$ & $60(100)$ \\
\hline \multicolumn{4}{|l|}{ Percepção da saúde } \\
\hline Muito boa / Boa & $23(76,6) *$ & $13(43,3)$ & 36 \\
\hline Regular & $5(16,6)$ & $14(46,6)$ & 19 \\
\hline Ruim / Muito ruim & $2(6,6)$ & $3(10)$ & 5 \\
\hline
\end{tabular}

${ }^{*}$ p-valor $<0,05$. Teste Qui quadrado.

era casada. A situação de viuvez pode estimular a pessoa a buscar integração com grupos de convivência e práticas de saúde, esportes e lazer. A prática de atividade física previne a dependência e estimula o bem-estar; condição que interfere positivamente na qualidade de vida, autoimagem e autoestima de idosas ${ }^{10}$. Uma pesquisa qualitativa realizada com idosas viúvas verificou que as atividades sociais se sobressaíram mais do que qualquer outro fator (como família ou novo companheiro) ${ }^{11}$. O grupo de Lian Gong é também espaço de relações interpessoais ${ }^{12}$ e a busca de atividades sociais pela condição de viuvez pode estar relacionada à maior concentração de idosas viúvas neste grupo.
Neste estudo, observou-se que os indivíduos ativos apresentaram uma percepção mais positiva sobre sua saúde. É provável que o maior número de relatos de percepção positiva para os ativos deva-se ao incentivo, existente na prática do Lian Gong, para o trabalho sobre a saúde numa perspectiva holística, que compreenda a qualidade de vida social, mental e espiritual ${ }^{5}$. Entre os indivíduos ativos, a percepção positiva da própria saúde esteve aliada a outros achados, como um menor consumo diário médio de medicamentos e uma menor sensação de dificuldade para realizar atividades devido à dor.

Nos idosos, sabe-se que os efeitos da atividade física sobre sua qualidade de vida e percepção 
Tabela 2. Aspectos relacionados aos agravos da saúde dos indivíduos. Campinas, SP, 2012.

\begin{tabular}{|c|c|c|c|}
\hline Categorias & $\begin{array}{c}\text { Ativos } \\
\mathbf{n}(\%)\end{array}$ & $\begin{array}{c}\text { Sedentários } \\
\mathbf{n}(\%)\end{array}$ & $\begin{array}{c}\text { Total } \\
\mathbf{n}\end{array}$ \\
\hline \multicolumn{4}{|l|}{ Quantidade de problemas de saúde citados } \\
\hline $0-1$ & $11(36,6)$ & $7(23,3)$ & 18 \\
\hline $2-3$ & $15(50)$ & $20(66,6)$ & 35 \\
\hline $4-5$ & $4(13,3)$ & $3(10)$ & 7 \\
\hline \multicolumn{4}{|l|}{ Categorias de problemas de saúde citados } \\
\hline Cardiovascular & 14 & 24 & 38 \\
\hline Metabólico & 16 & 13 & 29 \\
\hline Osteoarticulares & 13 & 15 & 28 \\
\hline Psiquiátrico & 1 & 3 & 4 \\
\hline Gastrintestinal & 7 & 3 & 10 \\
\hline Hepático & 2 & 2 & 4 \\
\hline Respiratório & 4 & 4 & 8 \\
\hline Outros & 8 & 8 & 16 \\
\hline Total & 65 & 72 & 137 \\
\hline \multicolumn{4}{|l|}{ Quantidade de medicamentos utilizados/dia } \\
\hline$<5$ medicamentos & $27(90 \%)$ & $17(56,7 \%)$ & $44(73,3 \%)$ \\
\hline$\geq 5$ medicamentos & $1(3,3 \%)$ & $9(30 \%)$ & $10(16,7 \%)$ \\
\hline Não souberam informar & $2(6,6 \%)$ & $4(13,3 \%)$ & $6(10 \%)$ \\
\hline \multicolumn{4}{|l|}{ Classificação ATC (nível 1) } \\
\hline Grupo A (Aparelho digestivo e metabolismo) & $22(29,7 \%)$ & $18(20,2 \%)$ & $40(24,5 \%)$ \\
\hline Grupo B (Sangue e órgãos hematopoiéticos) & $4(5,4 \%)$ & $7(7,9 \%)$ & $11(6,7 \%)$ \\
\hline Grupo C (Aparelho cardiovascular) & $30(40,5 \%)$ & $37(41,5 \%)$ & $67(41,1 \%)$ \\
\hline Grupo H (Preparações hormonais sistêmicas) & $3(4,1 \%)$ & $2(2,3 \%)$ & $5(3,1 \%)$ \\
\hline Grupo N (Sistema nervoso) & $5(6,8 \%)$ & $17(19,1 \%)$ & $22(13,5 \%)$ \\
\hline Grupo V (Vários) & $10(13,5 \%)$ & $8(9 \%)$ & $18(11,1 \%)$ \\
\hline Total & $74(100 \%)$ & $89(100 \%)$ & $163(100 \%)$ \\
\hline \multicolumn{4}{|l|}{ Quedas nos últimos 5 anos } \\
\hline Sim & $20(66,6)^{*}$ & $12(40)$ & $32(53,3 \%)$ \\
\hline Não & $10(33,3)$ & $18(60)$ & $28(46,7 \%)$ \\
\hline
\end{tabular}

${ }^{*}$ p-valor $<0,05$. Teste Qui quadrado.

da saúde reduzem a taxa de morbidade entre estes indivíduos ${ }^{13}$. Considerando que as doenças crônicas representam importante problema de saúde pública nesta população, os resultados do presente estudo corroboram sua presença em ambos os grupos de participantes, quando relataram suas principais causas de morbidade: cardiovascular, metabólica e osteoarticular.

Nesta perspectiva, os idosos sedentários faziam uso de mais medicamentos por dia (cinco ou mais), sendo que os mais citados foram os de ação cardiovascular. A frequência no uso de medicamentos de ação no sistema nervoso foi também maior neste grupo, na comparação com o grupo ativo.

Sendo a prática de atividades aeróbicas associada à redução do risco cardiovascular, do peso corporal, das taxas de colesterol sérico e da pressão $\operatorname{arterial}^{13}$, não é surpresa que o grupo de indivíduos ativos deste estudo tenha citado menos problemas cardiovasculares e menor uso de medicamentos.

Ainda que a literatura aponte que a atividade física na terceira idade reduz o risco de queda ${ }^{14,15}$, nesta amostra o número de quedas entre os indivíduos ativos foi estatisticamente maior. O risco de quedas entre idosos pode estar relacionado a fatores intrínsecos (medicamentos de ação cardiovascular; uso de quatro ou mais medicamentos; condições médicas específicas; osteoporose; diabetes) e/ou extrínsecos (riscos ambientais) ${ }^{16}$. Acredita-se que os indivíduos ativos se expõem mais aos riscos extrínsecos, já que relatam menos impedimentos relacionados à sensação dolorosa; assim, é possível que este grupo sofra mais quedas justamente por ser mais ativo, expor-se mais, e circular mais em ambientes pouco seguros. Este achado aponta para a necessidade de outras in- 
Tabela 3. Frequência de respostas positivas do Questionário Nórdico de Sintomas Osteomusculares para idosos sedentários e ativos. Campinas, SP, 2012.

\begin{tabular}{ccccccccc}
\hline \multirow{2}{*}{ Região da dor } & \multicolumn{2}{c}{$\begin{array}{c}\text { Dor nos últimos } \\
\mathbf{1 2} \text { meses }\end{array}$} & \multicolumn{2}{c}{$\begin{array}{c}\text { Viu-se impedido } \\
\text { de realizar atividade }\end{array}$} & $\begin{array}{c}\text { Buscou } \\
\text { prof. saúde }\end{array}$ & $\begin{array}{c}\text { Teve problema nos } \\
\text { últimos 7 dias }\end{array}$ \\
\cline { 2 - 9 } & $\begin{array}{c}\text { Ativo } \\
\mathbf{n}(\%)\end{array}$ & $\begin{array}{c}\text { Sed. } \\
\mathbf{n}(\%)\end{array}$ & $\begin{array}{c}\text { Ativo } \\
\mathbf{n}(\%)\end{array}$ & $\begin{array}{c}\text { Sed. } \\
\mathbf{n}(\%)\end{array}$ & $\begin{array}{c}\text { Ativo } \\
\mathbf{n}(\%)\end{array}$ & $\begin{array}{c}\text { Sed. } \\
\mathbf{n}(\%)\end{array}$ & $\begin{array}{c}\text { Ativo } \\
\mathbf{n}(\%)\end{array}$ & $\begin{array}{c}\text { Sed. } \\
\mathbf{n}(\%)\end{array}$ \\
\hline Pescoço & $17(56,6)$ & $12(40)$ & $9(30)$ & $12(40)$ & $13(43,3)$ & $11(36,6)$ & $13(43,3)$ & $8(26,6)$ \\
Ombro & $12(40)$ & $15(50)$ & $9(30)$ & $12(40)$ & $8(26,6)$ & $12(40)$ & $8(26,6)$ & $10(33,3)$ \\
Parte sup. costas & $10(33,3)$ & $11(36,6)$ & $6(20)$ & $9(30)$ & $6(20)$ & $8(26,6)$ & $7(23,3)$ & $4(13,3)$ \\
Cotovelo & $5(16,6)$ & $6(20)$ & $3(10)$ & $5(16,6)$ & $3(10)$ & $6(20)$ & $2(6,6)$ & $3(10)$ \\
Punho/mão & $14(46,6)$ & $19(63,3)$ & $5(16,6)$ & $10(33,3)$ & $9(30)$ & $11(36,6)$ & $9(30)$ & $8(26,6)$ \\
Parte inf. costas & $23(76,6)$ & $22(73,3)$ & $11(36,6)^{*}$ & $19(63,3)$ & $15(50)$ & $14(46,6)$ & $12(40)$ & $13(43,3)$ \\
Quadril/coxa & $8(26,6)$ & $13(43,3)$ & $5(16,6)$ & $9(30)$ & $6(20)$ & $11(36,6)$ & $4(13,3)$ & $11(36,6)$ \\
Joelho & $14(46,6)$ & $15(50)$ & $11(36,6)$ & $9(30)$ & $11(36,6)$ & $11(36,6)$ & $10(33,3)$ & $10(33,3)$ \\
Tornozelo/pé & $14(46,6)$ & $15(50)$ & $6(20)$ & $9(30)$ & $7(23,3)$ & $8(26,6)$ & $8(26,6)$ & $5(16,6)$ \\
\hline
\end{tabular}

Sed.: Sedentário; prof.:profissional; inf.: inferior; sup.: superior. ${ }^{*}$ p-valor $<0,05$. Teste Qui quadrado.

vestigações, bem como a construção e o planejamento de políticas públicas pertinentes. Particularmente na realidade estudada, são frequentes as reclamações de desatenção às necessidades dos idosos, seja nos transportes públicos, no calçamento das vias públicas, nas sinalizações de trânsito, dentre outros.

Desde o final do século passado e o início deste século a rede de atenção à saúde em Campinas conta com experiências exitosas de práticas integrativas e complementares, e vem se estruturando de modo a abarcar as ações de promoção da saúde ${ }^{3}$. Estas experiências acumuladas permitem indicar que o Lian Gong é uma das práticas a serem desenvolvidas com vistas à saúde, em especial dos longevos.

A predominância quase absoluta das mulheres nos grupos deve-se à maior frequência destas nos serviços de saúde, bem como a disposição que mostram para atividades coletivas e sociais. Destaca-se que, na América Latina, há considerável diferença entre homens e mulheres quanto a expectativa de vida e anos livres de adoecimento: os homens adoecem e morrem mais cedo, principalmente em populações mais pobres ${ }^{17}$.

Em relação aos relatos de sintomas osteomusculares e impedimentos para atividades de vida diária, observou-se que a quantidade de dores citadas por ambos os grupos é muito próxima, e que ambos os grupos apresentaram mais semelhanças que diferenças, exceto pelo fato de que as idosas ativas, apesar da dor, relatavam que se sentiam bem menos impedidas de realizar suas atividades.

Outro aspecto que merece destaque é a associação entre os fenômenos dolorosos e a convivência cotidiana ou o morar só. Num estudo sobre a prevalência de relatos dolorosos em 157 professores de uma cidade do interior de São Paulo, que também fez uso do questionário nórdico de sintomas osteomusculares para coleta de dados, obteve-se alta incidência de dor em punhos/mãos $(43,9 \%$ dos professores participantes). Este achado associou-se aos professores que não mantinham união estável. No olhar das pesquisadoras, como a maior parte da amostra também era do sexo feminino, o fato de não ter companheiro fixo acarretaria maiores responsabilidades às mulheres, principalmente econômicas, levando-as a trabalhar mais, e expondo-se ao estresse físico e psicológico ${ }^{18}$.

No nosso estudo detectou-se associação entre manter união estável (ou passar a maior parte do dia acompanhada) e não comprometer a mo- 
vimentação do pescoço nem apresentar dor na articulação do cotovelo. Por outro lado, passar a maior parte do dia sozinho associou-se a uma maior frequência de dor na região lombar. Estas associações são de difícil explicação; podem estar relacionadas ao estresse e esforço físico resultante da vida solitária, mas pedem investigações mais detalhadas e uma amostragem mais numerosa.

Um último aspecto diz respeito à relação do desenvolvimento de práticas de atividades físicas na atenção básica e o desenvolvimento de políticas públicas saudáveis para idosos, uma vez que se trata de um tema atual e global; tanto no Brasil $^{19}$ como na China ${ }^{20}$, as políticas públicas voltadas à saúde de idosos carecem de maior incremento, envolvimento e participação de uma série de sujeitos: aqueles diretamente interessados - os próprios idosos - os gestores e os profissionais da saúde.

A análise dos dados permite concluir que tanto os idosos ativos como os sedentários apresentaram relatos de presença de dor equiparados em quantidade; contudo, entre os indivíduos ativos houve menor consumo de medicamentos, e uma percepção mais positiva da sua própria saúde, com destaque para a menor limitação de movimentos na realização de atividades de vida diária. Não obstante, o fato de este estudo ter sido con- duzido utilizando amostra por conveniência pode representar uma limitação à validade externa. Mais pesquisas são necessárias para corroborar os achados que se definem como introdutórios.

\section{Considerações finais}

O trabalho no grupo de Lian Gong tem apresentado resultados muito significativos para a saúde, o empoderamento e o bem-estar dos idosos. A importância do grupo de convivência, a troca de experiências e o maior contato com o serviço e os profissionais de saúde contribuem para a saúde e autonomia destes indivíduos. Ficar sozinho e morar sozinho apresentaram importante relação com dores osteomusculares. São necessários mais estudos para entender essas relações, de forma a promover as práticas integrativas nos ambientes dos serviços de atenção básica, mormente na população idosa. É primordial que a equipe de saúde contribua para prevenir os agravos característicos do envelhecimento, ampliando o impacto das ações de saúde, a adesão aos grupos de atividades físicas e, por fim, obtendo bons resultados por meio de melhores práticas de cuidado e políticas de promoção à saúde.

\section{Colaboradores}

VCD Bobbo contribuiu na pesquisa, redação, edição e revisão do manuscrito; DD Trevisan e MCE Amaral contribuíram na discussão, redação, edição e revisão do manuscrito; e EM Silva contribuiu na orientação da pesquisa, discussão, redação, edição e revisão do manuscrito. 


\section{Referências}

1. United Nations (UN). World Population Ageing 2015. New York: UN; 2015.

2. World Health Organization (WHO). Noncommunicable diseases country profiles 2014. Geneva: WHO; 2014.

3. Nagai SC, Queiroz MS. Medicina complementar e alternativa na rede básica de serviços de saúde: uma aproximação qualitativa. Cien Saude Colet 2011; 16(3):1793-1800.

4. Miranda VS, Carvalho VBF, Machado LAC, Dias JMD. Prevalence of chronic musculoskeletal disorders in elderly Brazilians: a systematic review of the literature. BMC Musculoskeletal Disorders 2012; 13:82.

5. Souza JORL, Costa LS, Botecchia TE, Eufrásio S, Leite NM, Silva AL, Kozasa EH. Lian Gong: prática corporal chinesa e sua relação com a qualidade de vida em idosos. Saúde Coletiva 2010; 7(43):213-215.

6. Lee ML. Lian Gong em 18 terapias: forjando um corpo saudável. Ginástica chinesa do Dr. Zhuang Yuen Ming. 6a ed. São Paulo: Pensamento; 2011.

7. Brasil. Ministério da Saúde (MS). Caderneta de Saúde da Pessoas Idosa. Brasília: MS; 2010.

8. Barros ENC, Alexandre NMC. Cross-cultural adaptation of the Nordic musculoskeletal questionnaire. International Nursing Review 2003; 5(2):101-108.

9. World Health Organization (WHO). Collaborating Center for Drug Statistics Methodology. ATC/DDD Index (2016). Oslo: WHO; 2016.

10. Benedetti TB, Petroski EL, Gonçalves LT. Exercícios físicos, auto-imagem e auto-estima em idosos asilados. Rev. Bras. Cineantropom. Desempenho Hum. 2003; 5(2):69-74.

11. Rocha C, Gobbi I, Mazzarino M, Krabbe S, Areosa SVC. Como mulheres viúvas de terceira idade encaram a perda do companheiro. Revista Brasileira de Ciências do Envelhecimento Humano 2005; 2(2):65-73.

12. Nascimento ES, Branco MPFC, Moreira AKF, Hazime FA. Os efeitos do Lian Gong em hipertensos assistidos em unidade de saúde da família do município de Parnaíba, Piauí. Rev. Bras. Promoç. Saúde 2012; 25(4):435444.

13. Fries JF. Physical activity, the compression of morbidity, and the health of the elderly. J R Soc Med 1996; 89(2):64-68.
14. Gregg EW, Pereira MA, Caspersen CJ. Physical Activity, Falls, and Fractures Among Older Adults: A Review of the Epidemiologic Evidence. J Am Geriatr Soc 2000; 48(8):883-893.

15. El-Khoury F, Cassou B, Charles MA, Dargent-Molina P. The effect of fall prevention exercise programmes on fall induced injuries in community dwelling older adults: systematic review and meta-analysis of randomised controlled trials. BMJ 2013; 347:f6234

16. Khan KM, Liu-Ambrose T, Donaldson MG, McKay HA. Physical activity to prevent falls in older people: time to intervene in high risk groups using falls as an outcome. Br J Sports Med 2001; 35(3):144-145.

17. Haeberer M, Noguer I, Mújica OJ. Desigualdades educacionales en mortalidad y supervivencia de mujeres y hombres de las Américas, 1990-2010. Rev. Panam. Salud Publica 2015; 38(2):89-95.

18. Carvalho AJFP, Alexandre NMC. Sintomas osteomusculares em professores do ensino fundamental. Rev. Bras. Fisioter. 2006; 10(1):35-41.

19. Oliveira DC, Silva EM. Cuidado ao idoso em Campinas -SP: estudo qualitativo com gestores e representantes políticos. Rev. Bras. enferm. 2012; 65(6):942-949.

20. Ngai TFL, Chun Man YV, Yin TWL, Han CJ, Wai-yee CJ, Shing WTK. Nurses' knowledge of universal health coverage for inclusive and sustainable elderly care services. Rev. Latino-Am. Enfermagem 2016; 24:e2670.

Artigo apresentado em 27/04/2016

Aprovado em 08/07/2016

Versão final apresentada em 10/07/2016 